\title{
Suppression of Rcn3 in the alveolar epithelial cells may have beneficial effect against COPD
}

\section{Qianyu Zhang}

Beijing Chaoyang Hospital

\section{Tong Wang}

Beijing Chaoyang Hospital

Jiawei Jin

Beijing Chaoyang Hospital

Xiaoqian Shi

Beijing Chaoyang Hospital

\section{Zhenru Ma}

Beijing Chaoyang Hospital

\section{Runlin Z. Ma}

Institute of Genetics and Developmental Biology Chinese Academy of Sciences

Qiuhong Fang ( $\sim$ fangmaster123@163.com )

Beijing Chaoyang Hospital https://orcid.org/0000-0003-3012-8008

\section{Research}

Keywords: COPD, emphysema, Rcn3, type II alveolar epithelial cell

Posted Date: April 6th, 2020

DOI: https://doi.org/10.21203/rs.3.rs-20953/v1

License: (c) (i) This work is licensed under a Creative Commons Attribution 4.0 International License.

Read Full License 


\section{Abstract}

Background: Chronic obstructive pulmonary disease (COPD) is a highly prevalent lung disease worldwide and imposes increasing disease burdens globally. COPD is characterized by irreversible airflow obstruction. Emphysema is one of the primary pathological features causing the irreversible decline of pulmonary function, while the precise mechanisms behind emphysema remain unclear. Reticulocalbin 3 (Rcn3) is an endoplasmic reticulum (ER) lumen protein localized in the secretory pathway of living cells. We have reported that Rcn3 in type II alveolar epithelial cell (AECIIs) plays a critical role in perinatal lung development and bleomycin-induced lung injury-repair. Since is associated with alveolar epithelial disruption, Rcn3 might be involved in the development of emphysema during COPD.

Materials and Methods: We examined Rcn3 expression in lung specimens from patients (44 COPD patients and 26 non-COPD control patients) undergoing lung lobectomy or pneumonectomy. Mouse models of COPD and emphysema were established by cigarette smoke (CS) exposure and intratracheal installation of pancreatic porcine elastase (PPE), respectively. Rcn3 expression was detected in the lung tissues from these mice. Furthermore, conditional knockout (CKO) mice with Rcn3 deletion specific to AEClls, were used to explore the role of Rcn3 in PPE-induced emphysema progression. Rcn3 protein expression in lung tissues were evaluated by Western blot and immunohistochemistry. Rcn3 mRNA expression in lung tissues was detected by qPCR.

Results : Compared with non-COPD patients, Rcn3 expression was significantly increased in the lung specimens from COPD patients. Rcn3 expression was also significantly up-regulated in the lung tissues from COPD mice and emphysematous mice. Moreover, selective ablation of Rcn3 in AEC Ils significantly alleviated severity of the mouse emphysema in response to intratracheal installation of PPE.

Conclusions: Our data, for the first time, indicated that Rcn3 in AEClls might play a role in modulating the progression of emphysema, and thus be involved in the development of COPD. Suppression of Rcn3 expression in AEClls may have a beneficial effect on COPD.

This work was supported by National Natural Science Foundation of China (No. 81641004).

\section{Introduction}

Chronic Obstructive Pulmonary Disease (COPD) is a highly prevalent lung disease worldwide characterized by persistent and progressive airflow limitation[1]. The morbidity and mortality of COPD have gone up dramatically in the past decades, and it will become the third leading cause of death by 2020. Cigarette smoke (CS) is associated with COPD as the major risk factor of it[2]. Chronic bronchitis and emphysema are the main pathological features of COPD[3]. Emphysema is characterized by the enlargement of air space and the destruction of the parenchymal structure leading to progressive airflow limitation[1, 4]. The effective treatment for emphysema is limited, due to the insufficient knowledge regarding alveolar structural recovery and reconstruction in emphysema. 
Reticulocalbin3 (Rcn3) is a $\mathrm{Ca}^{2+}$-binding ER lumen protein, localized to the secretory pathway[5]. It belongs to the Cab45/Rcn/ERC45/Calumenin family[6], the biological function of which is still far from clear. We have previously reported that Rcn3 was expressed in a variety of mouse tissues with the highest expression in the lung[7]. Consistently, high expression of Rcn3 in human lung tissue has also been reported by Hou Yu et al[8]. We further revealed that Rcn3 knockout mice died from neonatal respiratory distress due to the impaired maturation of alveolar epithelial type II cells (AECIIs), suggesting its potential role in regulating AECIls function[7]. In addition, we demonstrated that the selective deletion of Rcn3 in AEClls exacerbated bleomycin-induced lung fibrosis[9]. We therefore hypothesized that Rcn3 might be involved in the development of emphysema through regulating AEClls function, as AEClls play a vital role in the pathogenesis of emphysema.

In this study, we first investigated the expression of Rcn3 in the lung specimens from COPD patients and CS-exposed mice. Rcn3 expression in the elastase-induced mouse model of emphysema was then evaluated. Furthermore, the potential effect of Rcn3 on the development of emphysema was studied by selectively knockout Rcn3 in the AEClls. We demonstrated a marked upregulation of Rcn3 expression in the lung tissues from COPD patients and COPD mice. We further found that a higher level of Rcn3 was detected in the lung tissues from the emphysema mice, and that selective deletion of Rcn3 was able to attenuate the degree of emphysematous changes. Our findings revealed for the first time that elevated Rcn3 in the lung was able to facilitate emphysema progression, and by which mechanism, Rcn3 might participate in the development of COPD.

\section{Materials And Methods}

\subsection{The collection of surgically resected specimens}

The human lung specimens were obtained from resected lung cancer within 30 minutes after resection from the Department of Thoracic Surgery, Beijing Shijitan Hospital between September 2013 and July 2015. The lung tissues (approximately $0.5 \mathrm{~cm}-1 \mathrm{~cm}^{3}$ ) were collected at least $10 \mathrm{~cm}$ away from the tumor edge. Pulmonary function test was performed in all patients. Based on the forced expiratory volume in 1second to forced vital capacity $($ FEV1/FVC) $<0.70[10]$, patients were assigned into the COPD group and the non-COPD group. The post-bronchodilator FEV1 percent predicted (FEV1\%) was used to measure the severity of airflow obstruction[1]. A total of 16 fresh lung-tissue samples were collected (10 cases of COPD, 6 cases of non-COPD). A total of 55 paraffin-embedded samples were collected (34 of the COPD, 21 of the non-COPD). A signed Informed Consent was obtained from each patient. The study was approved by the Ethics Committee for the Clinical Research of the Beijing Shijitan Hospital.

\subsection{Animals}

C57BL/6 mice (6 weeks old) were purchased from Beijing Vital River Laboratory Animal Technology Co., Lid. The doxycycline-induced AECIl-selective Rcn3 deletion (CKO) mice were established and identified by Runlin Z. Ma Lab in the Institute of Genetics and Developmental Biology, Chinese Academy of 
Sciences[9]. All the animals were housed in a temperature- and humidity-controlled room with free access to water and standard laboratory food. All animal procedures were approved by the Animal Care and Ethics Committee of Beijing Chao-Yang Hospital and were performed following the guide for the Care and Use of Laboratory Animals of Beijing Chao-Yang Hospital.

Generation of the doxycycline-induced AECII-selective Rcn3 deletion (CKO) mice The transgenic female mice with the selective deletion of Rcn3 in AEClls were generated using the Cre/loxp method. The tet-on system (triple-transgenic SP-C-rtTA ${ }^{\mathrm{tg} /-} /(\text { tetO })_{7} \mathrm{CMV}-\mathrm{Cre}^{\mathrm{tg} /-} / \mathrm{Rcn}^{\mathrm{fl} / \mathrm{fl}}, \mathrm{CKO}$ mice) was used to ablate Rcn3 in adulthood by doxycycline (DOX) administration[9]. Control mice were the littermates. Two groups of mice both have DOX in the drinking water at the age of 8 weeks old, until they had $0.15 \mathrm{U}$ PPE /per mouse administration at the age of 10 -weeks. Four weeks later, the lung tissues were collected.

Cigarette smoke exposure to the mice Total of $32 \mathrm{C} 57 \mathrm{BL} / 6 \mathrm{~J}$ (8-week-old) male mice acclimated for 2 weeks before the experimental procedures, and then mice were randomly assigned into the following two groups ( $n=16$ per group): 1) Control; 2) CS (exposed to cigarette smoke) group. Smoke exposure was performed in an inhalation chamber (1.0 mg of nicotine, $10 \mathrm{mg}$ of tar and $13 \mathrm{mg}$ of carbon oxide). CS group mice were exposed daily to CS for 2 hours a day, for 24 weeks[11]. Control mice were exposed to room air. Total lung capacity (TLC) and the number of total inflammatory cells in bronchial alveolar lavage fluid (BALF) were counted on day 181[12], then the lung tissues of two groups of mice were collected. This experimental protocol was approved by the Animal Ethics Committee of Beijing ChaoYang Hospital.

Protease-induced COPD emphysema model C57BL/6 female mice (weight 20-25 g, age 8 weeks) were randomly assigned to the following groups: Control group mice were treated with saline solution $(0.9 \%$ $\mathrm{NaCl}, 60 \mathrm{ul}$ ) and porcine pancreatic elastase (PPE) groups were treated one-time intratracheally with PPE (E1250, Sigma-Aldrich, St. Louis, Mo, USA)[13]. Some of the PPE-treated mice were also treated with atorvastatin at three different doses: $0.2 \mathrm{U} \otimes 0.3 \mathrm{U} \otimes 0.4 \mathrm{U} /$ per mouse in $60 \mathrm{ul}$ of saline solution. All animals were euthanized with cervical dislocation after 4 weeks of PPE administration and lung tissues were collected.

\subsection{Lung histology and morphometry}

The right lower lobe was inflated by $10 \%$ phosphate-buffered formalin for at least $48 \mathrm{~h}$ before embedding in paraffin and then were sectioned at a thickness of $4 \mu \mathrm{m}$. For morphometric studies, sections were stained with hematoxylin and eosin (HE)[14]. To calculate the average interalveolar distance and the change in alveolar destruction, the mean linear intercept (MLI) was used as a modified method for morphometric assessment. A rectangle grid comprising of 20 lines were drawn across $100 \times$ magnification H\&E staining images $(590.8 \times 886.2 \mu \mathrm{m})$ and the MLI was determined by dividing the total length of these lines $(14179.2 \mu \mathrm{m})$ by the total number of alveolar wall and grid line interceptions ( 5 fields were assessed at random for each lung)[13]. 


\subsection{Immunohistochemistry (IHC) and mean optical density analysis}

The paraffin-embedded slides were dewaxed, hydrated, and the antigen retrieved with the sodium citrate method for 5 minutes at high pressure[15]. The slices were incubated in $3 \%$ hydrogen peroxide for 30 minutes and then were blocked for 30 minutes. The Anti-Ren3 antibody was applied overnight in a wet box at $4{ }^{\circ} \mathrm{C}$. Next day, after washing off the primary antibody, the secondary antibody (Zhongshan Golden Bridge, Beijing, China) was applied for 30 minutes. Expression of the RCN3 was visualized with DAB reagent (Zhongshan Golden Bridge, Beijing, China) after the secondary antibody was washed off. The nucleus was stained with hematoxylin and then the sections were dehydrated and covered[16].

The mean optical density of Rcn3 positive cells in IHC assay was calculated by a quantitative dynamic program analysis of Image Pro Plus. Three random views were selected for each $100 \times$ magnification IHC images. The optical density of immunohistochemical staining positive cells divided by the total area of cells in each view and the value represented as the mean optical density of Rcn3 positive cells[17].

\subsection{Western blotting}

Protein concentration was quantified by the BCA method (CW Biotech, Beijing, China) and $30 \mu \mathrm{g}$ of protein extracts from left lung tissue was separated by sodium dodecyl sulfate-polyacrylamide gel electrophoresis (8\%). After electrophoresis, proteins were transferred to an NC membrane. The membrane was blocked with $5 \%$ skim milk at room temperature for 1 hour. The membranes were probed via overnight incubation at $4{ }^{\circ} \mathrm{C}$ with each of mouse or rabbit antibodies raised against: 1$)$ RCN3 (1:500, Santa Cruz Biotechnology, Dallas, TX, USA); 2) GRP78 (1:2000, Santa Cruz Biotechnology, Dallas, TX, USA); 3) Cleaved Caspase-3 (1:1000, Cell Signaling Technology, USA); 4) $\beta$-actin (1:5000, Santa Cruz Biotechnology, Dallas, TX, USA); 5) $\beta$-Tubulin (1:5000, Yeasen, Shanghai, China); 6) GAPDH (1:5000, Santa Cruz Biotechnology, Dallas, TX, USA); at $4{ }^{\circ} \mathrm{C}$ overnight. After washing with TBST, secondary antibodies (Zhongshan Golden Bridge, Beijing, China) were applied at room temperature for 2 hours. Protein bands were visualized using ECL reagent. ImageJ software was used for subsequent quantification and statistical analysis.

\subsection{Quantitative real-time PCR}

Total RNA was extracted from the right upper lobe and middle lobe, using Trizol (Life Technology-Ambion, Grand Island, NY, USA). Reverse-transcribed cDNA was amplified with SYBER Green qPCR SuperMix (CW Biotech, Beijing, China). RLP19 was used as an internal control. The melting curve was observed to identify the amplification specificity. The $2^{-\Delta \Delta C t}$ method was used to calculate the experimental results of RT-PCR results. The $\mathrm{Ct}$ represented the number of gene amplification cycle when fluorescence threshold is reached. Each sample was assessed in triplicate and at least three times.

\subsection{Statistical analysis}


Statistical analysis was performed by GraghPad Prism 5. Statistical comparisons between two groups were performed using Student's t-test (unpaired, 2-tailed). Chi-square test for comparison of two rates or two constituent ratios in the analysis of Characteristics of the patients. Pearson linear correlation analysis is used to quantitatively describe the correlation between FEV1\%pred and Rcn3 expression in lungs of patients. A p value of $<0.05$ indicated a significant difference between the groups. Independent experiments were repeated at least in triplicate and quantitative data were presented as mean \pm SEM (Stand error of the mean).

\section{Results}

\subsection{Rcn3 expression in the lung tissues from COPD and non- COPD patients}

Total 70 lung specimens (44 from COPD patients and 26 from non-COPD I patients) were obtained. Compared with non-COPD group, patients with COPD exhibited lower FEV1\% of predicted $(64.07 \pm 2.477$ compared with 100.2 $\pm 2.847, p<0.001$, additional file 1$)$, and lower FEV1/FVC (60.95 \pm 1.119 compared with $82.26 \pm 0.99, p<0.001$, additional file 2 ). The clinical characteristics including age, gender, and smoking history were shown in additional file 3.

Immunhistochemical analysis on the lung tissue specimens showed the destruction of the alveolar walls and enlargement of the alveolar spaces corresponding with the changes of emphysema in COPD patients. In COPD patient MLI was 1.3-fold $(p<0.05)$ of that measured in control patients (Fig. 1A, 1C). $\mathrm{IHC}$ results demonstrated Rcn3 protein was distributed in airway cells, fibroblasts, endothelial cells, alveolar macrophages and alveolar cells in lung tissues in the lung specimens from either group (Fig. 1A). Mean optical density analysis revealed that Rcn3 expression was significantly higher in the COPD patients than that in the non-COPD control group patients ( $p<0.05$, Fig. 1B). Quantitative RT-PCR and Western blotting further indicated that mRNA expression and protein level of Rcn3 were significantly higher in the lung specimens from COPD patient than that from the controls $(p<0.05$, Fig. 1D, 1E).

Pearson correlation test revealed that mRNA or protein of Rcn3 expression was negatively correlated with FEV1\%pred $(r=-0.8053, p<0.05$ and $r=-0.6064, p<0.05$, respectively, Fig. 1F). In addition, both mRNA expression and protein level of GRP78 were consistently elevated in the lungs of COPD patients compared with control lung tissues (both $p<0.05$, Fig. 1D, 1E).

\subsection{Rcn3 expression in the lung tissues of COPD mice models}

To further characterize the role of Rcn3 in the pathogenesis of COPD, a well-established experimental model of COPD in response to chronic CS exposure was used in the current study. C57BL/6 mice displayed obvious damaged alveolar septal and enlarged space in response to CS chronically. MLI and total lung capacity (TLC) of CS-exposed mice were higher than those of the air-exposed ones (Fig. 2A and 
2B, Table 1), demonstrating the features of emphysema. Rcn3 expression was significantly upregulated in CS-exposed mice compared with that of control animal either by immunohistochemistry $(p=0.0036$, Fig. 2A, 2C) or by immunoblot ( $p=0.0419$, Fig. 2D, 2E). Consistently, chronic CS exposure caused a significant increase in Rcn3 mRNA expression in the mice lung tissues $(p=0.0122$, Fig. 2F). In addition, GRP78 expression was significantly increased in the lung tissues from COPD model mice compared with the control ones (Fig. 2D, 2E and 2F). The number of total inflammatory cells in bronchial alveolar lavage fluid (BALF) was higher in CS-exposed mice than that in air-exposed one (Table 1). There was no significant correlation between the cell number in BALF and Rcn3 expressions at either mRNA or protein levels $(r=0.2349, p=0.3481$ and $r=0.1169, p=6044$ respectively, Fig. $2 G)$ as determined by Pearson correlation coefficient analysis.

Table 1

The number of total cells in BALF and TLC in Control and COPD mice $(x \pm s, n=$ 16)

\begin{tabular}{|lll|}
\hline & Total Cell Count in BALF $\left(\times \mathbf{1 0}^{4} / \mathrm{L}\right)$ & Total Lung Capacity $(\mathrm{TLC}, \mathrm{ml})$ \\
\hline Control & $6.86 \pm 3.81$ & $1.34676 \pm 0.1586$ \\
\hline COPD & $12.22 \pm 6.25^{*}$ & $1.50316 \pm 0.0952^{*}$ \\
\hline Data presented as mean $\pm \mathrm{SEM}{ }^{*}{ }^{*} \mathrm{p}<0.05$, versus Control group \\
\hline
\end{tabular}

\subsection{Rcn3 expression in the lung tissues of the emphysema mice models}

Rcn3 expression was further examined in mouse model of emphysema induced by administration of PPE, which was established at day 28 of PPE instillation. Findings of HE staining showed that all the three concentrations $(0.2 \mathrm{U}, 0.3 \mathrm{U}$ and $0.4 \mathrm{U})$ of PPE resulted in prominent emphysematous changes, while the lungs from the sham mice kept intact alveolar septa with preserved air spaces. Among the three PPEtreatment groups, 0.3U-PPE-group presented the most severe emphysematous alteration (Fig. 3A), which was further confirmed by morphometry. In this regard, MLI of each PPE-treatment group $(56.10 \mu \mathrm{m}$, $98.23 \mu \mathrm{m}$ and $71,22 \mu \mathrm{m}$ for $0.2 \mathrm{U}-, 0.3 \mathrm{U}$ - and 0.4U-PPE groups, respectively) was larger than that of the control group $(40.58 \mu \mathrm{m})(\mathrm{p}<0.05, \mathrm{p}<0.001$ and $\mathrm{p}<0.001$ for $0.2 \mathrm{U}-, 0.3 \mathrm{U}$ - and $0.4 \mathrm{U}-\mathrm{PPE}$ groups, respectively). MLIs of the $0.2 \mathrm{U}$ - and $0.4 \mathrm{U}-\mathrm{PPE}$ groups were lower than that of $0.3 \mathrm{U}$-PPE group (both $\mathrm{p}<$ 0.001 , Fig. 3B). Rcn3 protein expression was significantly increased in each PPE-treated group compared to that of control animals, and the 0.3U-PPE group presented the highest RCN3 level (3.0-fold increase) among the three PPE experimental groups (Fig. 3C, 3D), which was in accordance with the observed histological alteration pattern and MLI measurement in the animals. In addition, mRNA expression of Rcn3 was significantly increased in the 0.3U-PPE-treatment mice, with a 3.42-fold increase, in comparison with the sham ones (Fig. 3E). 
In addition, protein levels of GRP78 and cleaved Caspase-3 were significantly increased in the three PPE experimental groups compared with that of the sham group, and the 0.3U-PPE group presented the highest level of cleaved Caspase-3 (Fig. $3 \mathrm{C}$ and D). Interestingly, compared to the groups of lower PPE concentrations, 0.4U-PPE-treatment augmented protein level of GRP78 (Fig. 3C, 3D). In addition, mRNA expressions of GRP78, MMP-9 and Col-1 were up-regulated in the 0.3U-PPE-treatment mice $(p<0.01, p<$ 0.05 and $p<0.05$, respectively, Fig. $3 E$ ) compared to the control mice.

\subsection{Selective deletion of Rcn3 in the mouse AEClls alleviated severity of elastase-induced emphysema}

To further investigate the role of Rcn3 in the pathogenesis of emphysema, AECII-selective Rcn3 knockout (CKO) mice were successfully established in our laboratory and used to induce mouse model of emphysema by PPE administration. Expression of mRNA as well as protein of Rcn3 were significantly reduced in whole lung homogenates from PPE-treated CKO mice compared with that of PPE-treated WT mice $(p<0.05$ in mRNA and $<0.01$ in protein, Fig. 4B, 4C). As presented in Fig. 4A, severity of emphysematous alteration was alleviated in the CKO mice compared to that in the wide type mice as evidenced by the MLI values ( $52.91 \pm 1.48 \mu \mathrm{m}$ of CKO mice vs $70.07 \pm 5.07 \mu \mathrm{m}$ of wild type mice, $\mathrm{n}=10 \mathrm{in}$ each group, $p<0.01$, Fig. 4A). Protein level of GRP78 was significantly decreased in PPE-treated CKO mice compared to the PPE-treated WT mice $(p<0.05)$, while no significant alteration of cleaved Caspase3 protein was found in two groups (Fig. 4B). Accordingly, mRNA expressions of GRP78, MMP-9 and Col-1 were significantly down-regulated in PPE-treated CKO mice lung (Fig. 4C).

\section{Discussion}

In the present study, we for the first time demonstrated that RCN3 expression was significantly increased in the lungs from COPD patients. Consistently, Rcn3 expression was unregulated in the lung tissues from both CS-exposed mouse model of COPD and elastase-induced emphysema mice. Furthermore, the selective ablation of Rcn3 in AEClls alleviated the degree of PPE-induced emphysema. These results indicate that Rcn3 expression in the lung AEClls may play a pivotal role in the pathogenesis and progression of emphysema, and thus might participate in the development of COPD.

In the current study, lung specimens were obtained from the patients experienced lobectomy or pneumonectomy for lung nodule, who were either COPD or non-COPD control subjects without any other airway and lung diseases except the lung nodule. Different degree of emphysematous alteration was observed in the lung of COPD patients, and was confirmed by larger MLI measurement in comparison with that of the control patients. Consistent with our previous report[8], Rcn3 express in human lung tissue was demonstrated, predominantly in the alveolar epithelial cells. Furthermore, Rcn3 expression was significantly higher in the emphysematous lungs from COPD patients compared to that from the control group, suggesting Rcn3 might be associated with COPD.

COPD was characterized by airflow limitation, which was reflected by FEV1\%pred from pulmonary function test[1]. Here, we demonstrated that protein and mRNA level of Ren3 in human lung was 
negatively correlated with the value of FEV1\%pred. Emphysema, one of the major pathological features of COPD, leads to the loss of lung elastic recoil, contributing partially to airflow limitation[18]. Cigarette smoke has been known to be the main cause of COPD[19] and Rabe et al have demonstrated that CSinduced animal model of COPD usually promotes morphological changes compatible with mild emphysema[20]. Consistently, in the current study, it was found that $97.7 \%$ of COPD patients were smokers or ex-smokers, while only $11.5 \%$ patients had smoking history in the control group. To further investigate the role of Rcn3 in the development of COPD, we established the mouse model of COPD by chronic cigarette smoke exposure, in which, obvious emphysema pattern and increased MLI were confirmed histologically. Consistent with the aforementioned findings in the patients, significant upregulation of Rcn3 protein and mRNA expression was confirmed in the lung tissues from COPD mice compared to that from the control group, suggesting Rcn3 may play a role in the pathogenesis of COPD in response to cigarette smoke exposure although no correlation was found between the cell number in the BALF and Rcn3 level in the lung of mice COPD model. .

The pathological feature of COPD is complicated by chronic bronchitis, small airway fibrosis and emphysema[4, 21]. To further investigate the role of Rcn3 in emphysema, the mouse model of emphysema by intra-trachea administration of PPE was used in the current study. PPE instillation induced more severe morphological alterations, corresponding to the histological feature of emphysema and bullae in lung, than that in chronic CS-exposure mice[22]. Similar to the results of Ip MP et al[23], the current study demonstrated that 0.4U PPE instillation produced less severe degree of emphysema than that of 0.3U PPE instillation. Nevertheless, Rcn3 expression was significantly increased in the emphysematous lungs induced by all the three concentrations of PPE $(0.2,0.3$, and $0.4 \mathrm{U})$ compared to the sham one, and the level of Rcn3 seemed to be correlated with the degree of emphysema with the highest Rcn3 expression corresponding to the most severe emphysematous alterations. The mechanisms behind emphysema formation were multifactorial. In this regard, proteolysis and oxidative injury were known to be involved in addition to the central role of inflammatory process[1]. The experimental mouse model of emphysema induced by administration of elastase was mainly based on the currently accepted hypothesis of protease-antiprotease imbalance[24], although the downstream pathway was not fully elucidated. Similar to the results of previous reports[25-27], elevated MMP-9 level was found in the PPEinduced emphysematous lungs along with augmented expression of collagen 1 in the current animal models, which was considered to result from over-synthesis of ineffective collagen[28]. Up-regulation of Rcn3 in the lungs from COPD patients as well as from animal models of cigarette smoke-induced COPD lungs and PPE-induced emphysematous lungs suggested that Rcn3 might promote the process of proteolytic injury.

Previous study has found that mice with whole body Ren3 knockout died after birth because of respiratory failure[7]. To further investigate the role of Rcn3 in pulmonary diseases, selective Rcn3 knockout in AEClls were successfully established in our laboratory, and have been employed to study the function of Rcn3 in the development of lung fibrosis[9]. We hereby established the PPE-induced emphysema animal model in the AECll-selective Rcn3 knockout mice to further explore if the increased Rcn3 expression in lung tissue affected the histological alterations of emphysema. Selective deletion of 
Rcn3 in the AEClls resulted in significant remission in severity of emphysematous changes in mice response to PPE instillation as evidenced by $26.4 \%$ reduction in MLI. In addition, while cleaved caspase-3 protein level was enhanced in PPE group compared to the control group, there was no significant difference in protein level of cleaved caspase-3 in emphysema mice lungs of AECII-selective Rcn3 ablation versus wild type mice, suggesting Rcn3 might not participate in apoptosis-related pathways of emphysema progression. However, MMP-9 and collagen 1 were down-regulated in the emphysema mice with AECII-selective Rcn3 ablation. These findings strongly suggested that Rcn3 may promote the development of emphysema and suppression of Rcn3 expression may have protective effect in COPD through ameliorating emphysematous alterations by modifying the responses to proteolytic injury.

An accumulation of misfolded and unfolded proteins in ER lumen under physiological or pathological stress results in ER stress[29]. To restore protein homeostasis, the unfolded protein response (UPR) is mediated and activated by ER membrane protein sensors, which were regulated by GRP78[30, 31]. Recently, it has been shown that ER stress was induced in mouse lungs in response to CS and the lungs of chronic smokers[32]. In the current study, we observed the upregulation of GRP78 in the lungs not only from COPD patients but also from mouse models of COPD and emphysema. In the PPE-induced emphysema mice, the lung expressed less GRP78 when Rcn3 was deleted selectively in the AECIls, which was in same direction with the changes of Rcn3 expression. Interfering with ER stress probably was involved in the biological mechanisms behind the process of emphysema regulated by Rcn3. This result seemed to conflict with the previous findings, in which lung specimens from the mouse model of lung fibrosis induced by bleomycin administration expressed higher level of GRP78 when Rcn3 was deleted selectively in AECIIs[9]. This is probably due to the difference of duration needed to establish each typical animal model, only 7 days for lung fibrosis formation after bleomycin challenge, while elastase-induced emphysema model experienced a more chronic process of 28 days after PPE instillation. Although the downstream signal transduction pathway of ER stress affected by Rcn3 is not clear, the present study revealed that Rcn3 deficiency in AEClls was able to stimulate repair process of lung, presumably by regulating MMP-9 and type I collagen production as aforementioned. Similarly, Jin et al. observed that Rcn3 deficiency in AEClls exacerbated lung fibrosis resulting from over activation of repair function[9]. An in vitro study[33]also demonstrated that fibroblasts isolated from the heart exhibited up-regulation of extracellular matrix production after Rnc3 knocked out, which implied that Rcn3 might participate in injury-repair process and thus affect tissue remodeling.

In conclusion, findings of the current study, for the first time, indicated that Rcn 3 might be involved in the development of COPD. Suppression of Rcn3 expression in the lung may have protective effect on COPD by attenuating the progression of emphysema. Additional biological function of Rcn3 on alveolar epithelial cells and lung fibroblasts and its mechanisms, however, remain to be further investigated.

\section{Abbreviations}

COPD Chronic obstructive pulmonary disease 
Rcn3 Reticulocalbin 3

ER endoplasmic reticulum

AEClls type II alveolar epithelial cell

CS cigarette smoke

PPE pancreatic porcine elastase

CKO mice conditional knockout (CKO) mice with Rcn3 deletion specific to AEClls

FEV1/FVC the forced expiratory volume in 1-second to forced vital capacity

FEV1\% The post-bronchodilator FEV1 percent predicted

TLC Total lung capacity

BALF bronchial alveolar lavage fluid

HE hematoxylin and eosin

MLI mean linear intercept

IHC Immunohistochemistry

\section{Declarations}

Ethics approval and consent to participate

Human data in the study has been performed in accordance with the Declaration of Helsinki. Ethical approval for the clinical study was obtained from the Research Ethics Committee for the Clinical Research of the Beijing Shijitan Hospital.

All animal procedures were approved by the Animal Care and Ethics Committee of Beijing Chao-Yang Hospital and were performed following the guide for the Care and Use of Laboratory Animals of Beijing Chao-Yang Hospital.

Competing interest

The authors declare that they have no competing interests.

Funding

This work was supported by National Natural Science Foundation of China (No. 81641004).

Authors' contributions

FQ and MR made the contribution on study conceptualization and methodology. JJ reviewed and edited the manuscript. ZQ was a major contributor in writing the manuscript. WT performed the data collection 
and analysis. All authors read and approved the final manuscript.

Acknowledgements

We thank Prof. Yu Kuang in China Agricultural University for providing guides to experimental methods.

Availability of data and materials

All data generated or analyzed during this study are included in this published article [and its supplementary information files].

Consent for publication

Not applicable.

Author details

1Department of Respiratory and Critical Care Medicine, Beijing Chaoyang Hospital, Capital Medical University, Beijing,100020, China. 2The Clinical Research Center, Beijing Chaoyang Hospital, Capital Medical University, Beijing, 100020, China. 3Institute of Genetics and Developmental Biology, Chinese Academy of Sciences $₫$ Beijing $₫ 100101 \otimes C h i n a$

\section{References}

1.

Singh D, Agusti A, Anzueto A, Barnes P, Bourbeau J, Celli B, Criner G, Frith P, Halpin D, Han M, et al: Global Strategy for the Diagnosis, Management, and Prevention of Chronic Obstructive Lung Disease: the GOLD science committee report 2019. 2019, 53.

2.

Am Miniño J, Xu. Control Kochanek Kd \%J National Vital Statistics Reports: From the Centers for Disease, Prevention National Center for Health Statistics, National Vital Statistics System: Deaths: preliminary data for 2008. 2010, 59:1-52.

3.

Cf Vogelmeier G, Criner F, Martínez A, Anzueto P, Barnes J, Bourbeau B, Celli R, Chen M, Decramer L.

Fabbri, et al: Global Strategy for the Diagnosis, Management, and Prevention of Chronic Obstructive Lung Disease 2017 Report: GOLD Executive Summary. 2017, 53:128-149.

4.

Stolz D, Barandun J, Borer H, Bridevaux P, Brun P, Brutsche M, Clarenbach C, Eich C, Fiechter R, Frey M, et al: Diagnosis, Prevention and Treatment of Stable COPD and Acute Exacerbations of COPD: The Swiss Recommendations 2018. 2018, 96:382-398.

5 .

News Honoré. B \%J Bioessays:, Reviews in Molecular Cellular, Biology Developmental: The rapidly expanding CREC protein family: members, localization, function, and role in disease. 2009, 31:262-277. 6.

Tsuji A, Kikuchi Y, Sato Y, Koide S, Yuasa K, Nagahama M, Matsuda Y. A proteomic approach reveals transient association of reticulocalbin-3, a novel member of the CREC family, with the precursor of 
subtilisin-like proprotein convertase, PACE4. Biochem J. 2006;396:51-9.

7.

Jin J, Li Y, Ren J, Lam SM, Zhang Y, Hou Y, Zhang X, Xu R, Shui G, Cell Ma Rz \%J American Journal of Respiratory, Biology Molecular: Neonatal Respiratory Failure with Retarded Perinatal Lung Maturation in Mice Caused by Reticulocalbin 3 Disruption. 2016, 54:410-423.

8.

Hou Y, Li Y, Gong F, Jin J, Huang A, Fang Q, Ma RZ. A Preliminary Study on RCN3 Protein Expression in Non-small Cell Lung Cancer. Clin Lab. 2016;62:293-300.

9 .

Jin J, Shi X, Li Y, Zhang Q, Guo Y, Li C, Tan P, Fang Q, Ma Y. Cell Ma Rz \%J American Journal of Respiratory, Biology Molecular: Reticulocalbin 3 Deficiency in Alveolar Epithelium Exacerbated Bleomycininduced Pulmonary Fibrosis. 2018, 59:320-333.

10.

JI López-Campos W, Tan. Respirology Soriano Jb \%J: Global burden of COPD. 2016, 21:14-23. 11.

Rashid K, Sundar I, Gerloff J, Li D, Reports Rahman I \%J Scientific: Lung cellular senescence is independent of aging in a mouse model of COPD/emphysema. 2018, 8:9023.

12.

Fuchimoto Y, Kanehiro A, Miyahara N, Koga H, Ikeda G, Waseda K, Tanimoto Y, Ueha S, Kataoka M. Ew Gelfand, et al: Requirement for chemokine receptor 5 in the development of allergen-induced airway hyperresponsiveness and inflammation. 2011, 45:1248-1255.

13.

Am Anciães C, Olivo C, Prado Kh, Kagohara SP, Tda Ht, Moriya T, Mauad. A Martins Mde, Clinics Lopes Fd $\% \mathrm{~J}$ : Respiratory mechanics do not always mirror pulmonary histological changes in emphysema. 2011, 66:1797-1803.

14.

Taguchi L, Pinheiro N, Olivo C, Choqueta-Toledo A, Grecco S, Lopes Fd, Caperuto L, Martins M. If Tiberio, No Câmara, et al: A flavanone from Baccharis retusa (Asteraceae) prevents elastase-induced emphysema in mice by regulating NF-KB, oxidative stress and metalloproteinases. 2015, 16:79.

15.

Zou Y, Li S, Zou W, Hu G, Zhou Y, Peng G, He F, Li B. One Ran P \%J Plos: Upregulation of gelatinases and epithelial-mesenchymal transition in small airway remodeling associated with chronic exposure to wood smoke. 2014, 9:e96708.

16.

Yang C, Li L, Guo J, Zhang W, Zhu W, Rao X, Huang W. \%J Pathology Research, Practice: Up-regulation of Pim-3 in Chronic Obstructive Pulmonary Disease (COPD) patients and its potential therapeutic role in COPD rat modeling. 2017, 213:322-326.

17.

Cai X, Ni X, Yi Y, He H, Wang J, Fu Y, Sun J, Zhou J, Cheng Y, Jin J, et al: Overexpression of CD39 in hepatocellular carcinoma is an independent indicator of poor outcome after radical resection. 2016, 
95:e4989.

18.

Cm Bidan A, Veldsink H, Meurs. Physiology Gosens R \%J Frontiers In: Airway and Extracellular Matrix Mechanics in COPD. 2015, 6:346.

19.

Cj, Murray. Medicine Lopez Ad \%J the New England Journal Of: Measuring the global burden of disease. 2013, 369:448-457.

20.

Kf Rabe S, Hurd A, Anzueto P, Barnes S, Buist P, Calverley Y, Fukuchi C, Jenkins R, Rodriguez-Roisin C, Van Weel, et al: Global strategy for the diagnosis, management, and prevention of chronic obstructive pulmonary disease: GOLD executive summary. 2007, 176:532-555.

21.

Zhou Y, Horowitz JC, Naba A, Ambalavanan N, Atabai K, Balestrini J, Bitterman PB, Corley RA, Ding BS, Engler AJ, et al. Extracellular matrix in lung development, homeostasis and disease. Matrix Biol. 2018;73:77-104.

22.

Rodrigues R, Olivo C, Lourenço Jd, Riane A, Cervilha D, Ito Jt. Ma Martins, Tisilogia Lopes Fdtqds \% J Jornal Brasileiro De Pneumologia: Publicacao Oficial Da Sociedade Brasileira De Pneumologia E: A murine model of elastase- and cigarette smoke-induced emphysema. 2017, 43:95-100. 23.

Mp Ip J, Kleinerman V, Ranga J, Sorensen. Disease Powers Jc \% J the American Review of Respiratory: The effects of small doses of oligopeptide elastase inhibitors on elastase-induced emphysema in hamsters: a dose-response study. 1981, 124:714-717.

24.

Boyer L, Plantier L, Dagouassat M, Lanone S, Goven D, Caramelle P, Berrehar F, Kerbrat S, Dinh-Xuan At, Crestani B, et al: Role of nitric oxide synthases in elastase-induced emphysema. 2011, 91:353-362. 25.

Lopes FD, Toledo AC, Olivo CR, Prado CM, Leick EA, Medeiros MC, Santos AB, Garippo A, Martins MA, Mauad T. A comparative study of extracellular matrix remodeling in two murine models of emphysema. Histol Histopathol. 2013;28:269-76.

26.

Lanone S, Zheng T, Zhu Z, Liu W, Lee C, Ma B, Chen Q, Homer R, Wang J, Rabach L, et al: Overlapping and enzyme-specific contributions of matrix metalloproteinases-9 and - 12 in IL-13-induced inflammation and remodeling. 2002, 110:463-474.

27.

Zheng T, Zhu Z, Wang Z, Homer R, Ma B, Riese R, Chapman H. Sd Shapiro, Investigation Elias Ja \%J the Journal of Clinical: Inducible targeting of IL-13 to the adult lung causes matrix metalloproteinase- and cathepsin-dependent emphysema. 2000, 106:1081-1093.

28. 
Shifren A, Society Mecham Rp \%J Proceedings of the American Thoracic: The stumbling block in lung repair of emphysema: elastic fiber assembly. 2006, 3:428-433.

29.

Barreiro E, Salazar-Degracia A, Sancho-Munoz A, Gea J. Endoplasmic reticulum stress and unfolded protein response profile in quadriceps of sarcopenic patients with respiratory diseases. J Cell Physiol. 2019;234:11315-29.

30.

Wang Y, Wu Z, Oncotarget Wang W, \%J. Inhibition of endoplasmic reticulum stress alleviates cigarette smoke-induced airway inflammation and emphysema. 2017, 8:77685-77695.

31.

Jorgensen E, Stinson A, Shan L, Yang J, Gietl D. Cancer Albino Ap \%J Bmc: Cigarette smoke induces endoplasmic reticulum stress and the unfolded protein response in normal and malignant human lung cells. 2008, 8:229.

32 .

Naiel S, Tat V, Padwal M, Vierhout M, Mekhael O, Yousof T, Ayoub A, Abed S, Dvorkin-Gheva A, Chest Ask K \%J: Protein Misfolding and ER stress in Chronic Lung Disease: Will Cell-Specific Targeting be the Key to the Cure? 2019.

33.

Martínez-Martínez E, Ibarrola J, Fernández-Celis A, Santamaria E, Fernández-Irigoyen J, Rossignol P, Jaisser F. Reports López-Andrés N \%J Scientific: Differential Proteomics Identifies Reticulocalbin-3 as a Novel Negative Mediator of Collagen Production in Human Cardiac Fibroblasts. 2017, 7:12192.

\section{Figures}


Figure 1
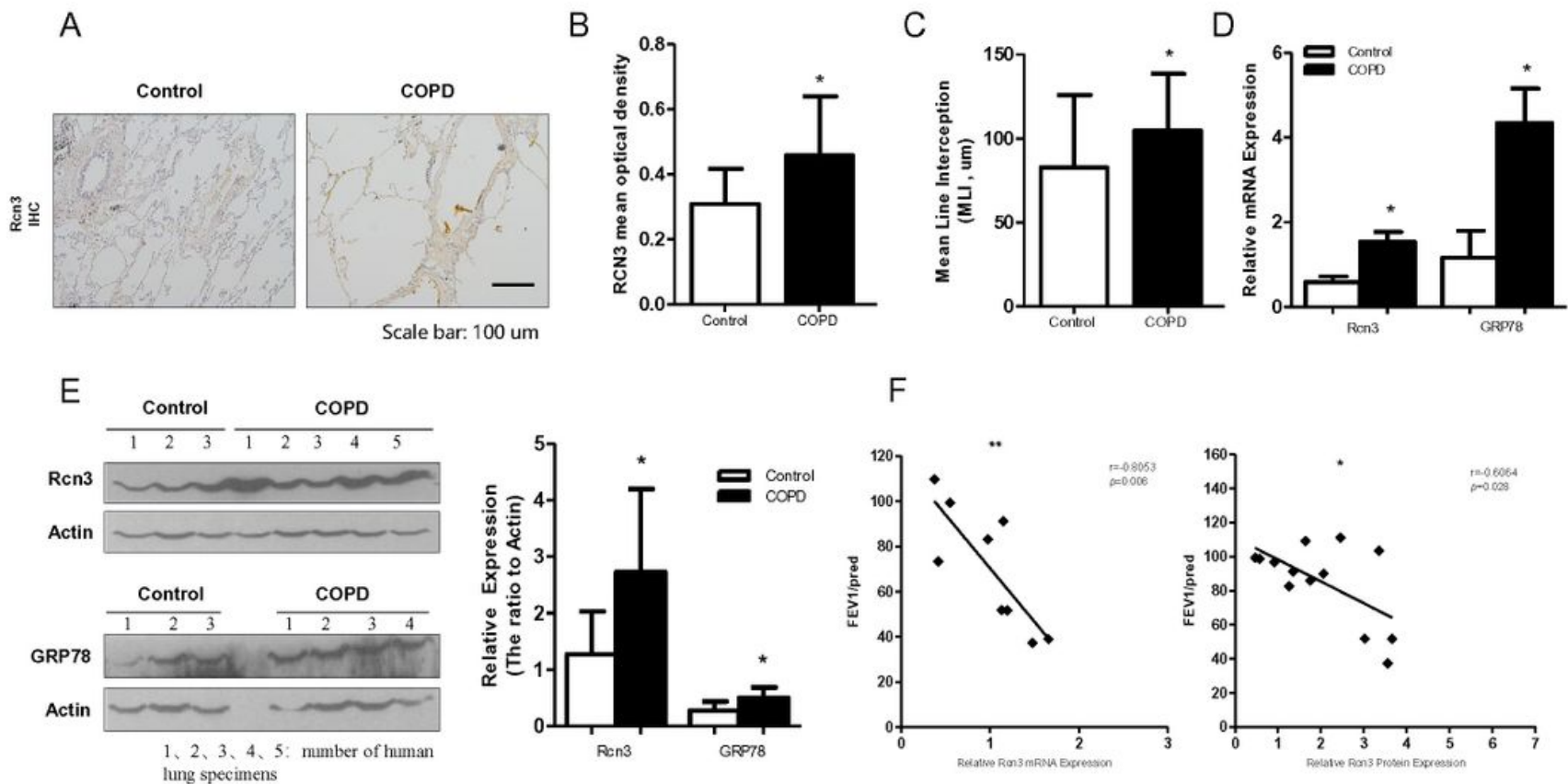

\section{Figure 1}

Rcn3 expression in lung tissues from COPD patients. Patients were divided into COPD group and Control group according to the pulmonary function test (FEV1/FVC). (A) IHC assays of Rcn3 from COPD group lungs and Control group lungs ; (B) Quantification of the mean optical density of Rcn3 expression in the lung tissues according to the Rcn3 IHC assays (3 random views, 100x amplification for one subject, $\mathrm{n}=36) ;(\mathrm{C}) \mathrm{MLI}$ was measured in IHC assay (0.3415 \pm 0.0315 vs.0.3462 $\pm 0.0362, \mathrm{n}=55, \mathrm{p}<0.05)$; (D) Qualitative RT-PCR analysis of the mRNA of Rcn3 and GRP78; the data are normalized to the RLP19 content and are analyzed by the 2- $\Delta \Delta \mathrm{Ct}$ method relative to the Control group. (E) The protein levels of Rcn3 and GRP78 are examined by Western blot analysis and the ratios to Actin presented by bar graph; (F) A negative correlation between the expression of Rcn3 mRNA and FEV1\%pred. $(r=-0.8053, p=0.008$, $n=9)$; a negative correlation between the expression of Rcn3 protein and FEV1\%pred. $(r=-0.6064, n=10$, $p=0.028$,). Data presented as mean $\pm S E M ;{ }^{*} p<0.05$, ${ }^{*} p<0.01$, versus littermate control in the same treatment group. Bar size: $100 \mu \mathrm{m}$. 
Figure 2

A

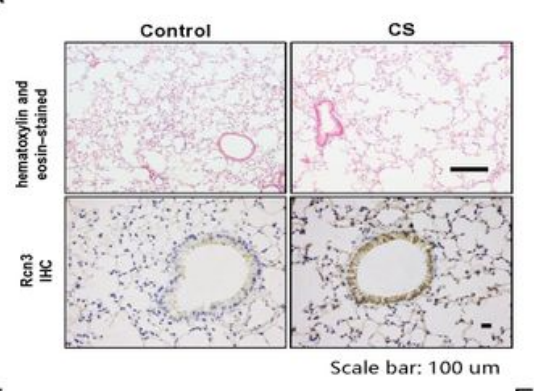

$\mathrm{E}$

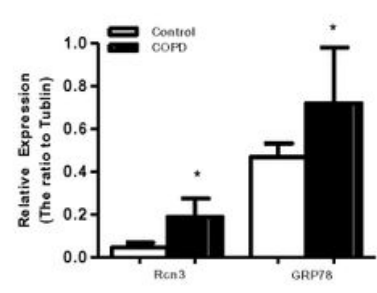

B

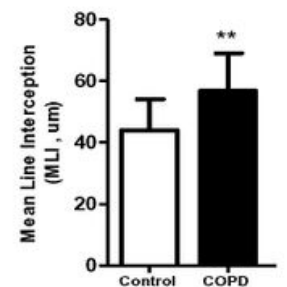

$\mathrm{F}$

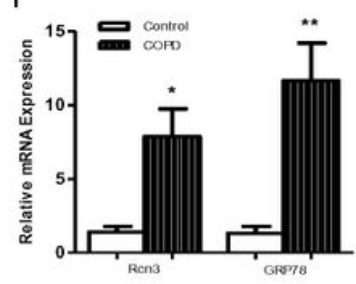

C
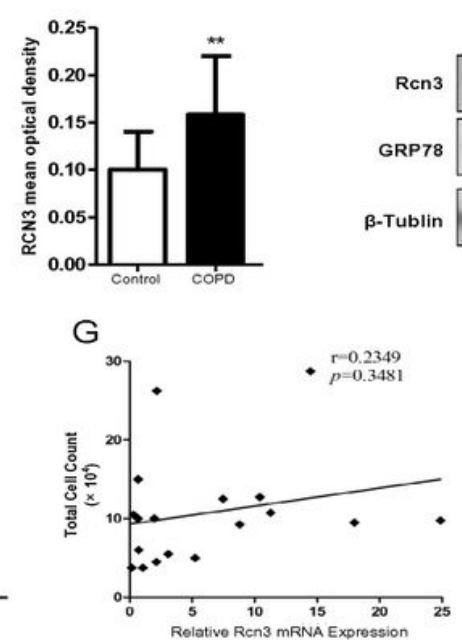

D
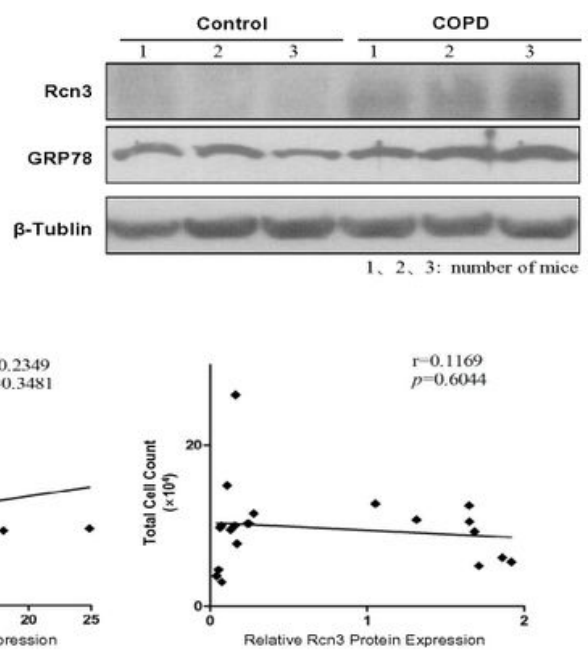

Figure 2

Rcn3 expression in lung tissues from CS-induced mouse model of COPD. C57BL/6J mice (8 weeks old) were exposed to CS for 6 months as COPD model mice. (A) Photomicrography of pulmonary parenchyma stained with HE (upper panels); IHC assays of Rcn3 (lower panels); (B) Morphometric analysis of the MLI $(n=32) ;(C)$ Quantification of the mean optical density of Rcn3 expression in the lung tissues according to the Rcn3 IHC assays ( $n=16$ ); (D) Representative WB for protein levels of Rcn3 and GRP78 in lungs. $\beta$ Tubulin was used as loading control; (E) Densitometric analysis of WB for Rcn3 and GRP78; (F) Qualitative RT-PCR analysis of the mRNA of Rcn3 and GRP78. The data are normalized to the RLP19 content; (G) The correlation analysis between cell number in the BALF and Rcn3 expression at both mRNA and protein levels $(r=0.2349, p=0.3481$ and $r=0.1169, p=6044$ respectively). Data presented as mean $\pm S E M ;{ }^{*} p<0.05,{ }^{*} p<0.01$, versus littermate control in the same treatment group. Bar size: $100 \mu m$. 
Figure 3

A

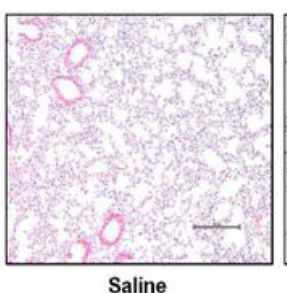

Saline

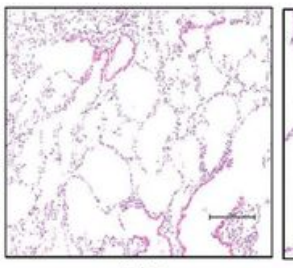

$0.2 \mathrm{U}$

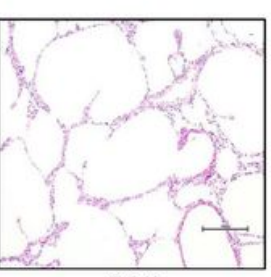

$0.3 \mathrm{U}$

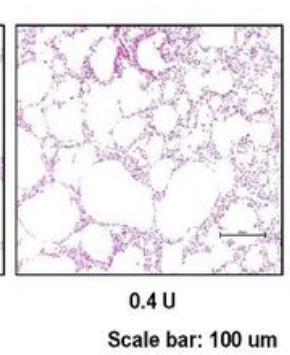

$\mathrm{E}$
C

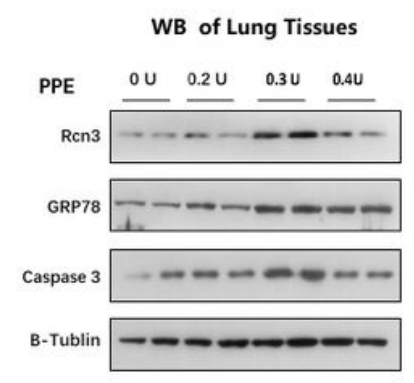

D

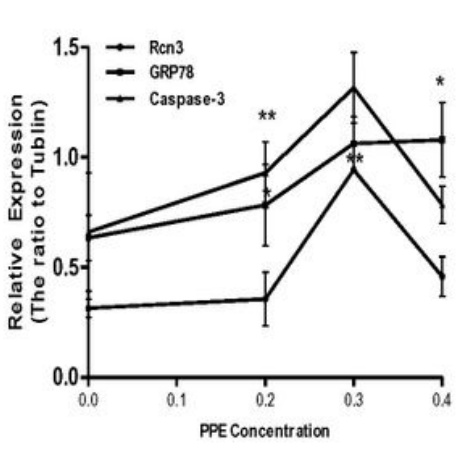

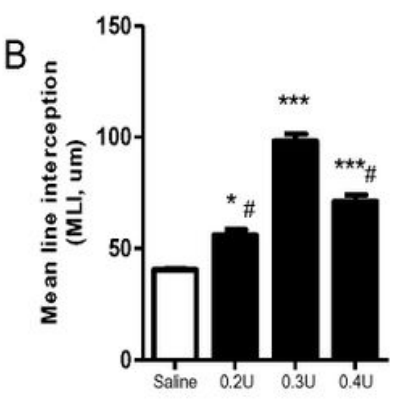

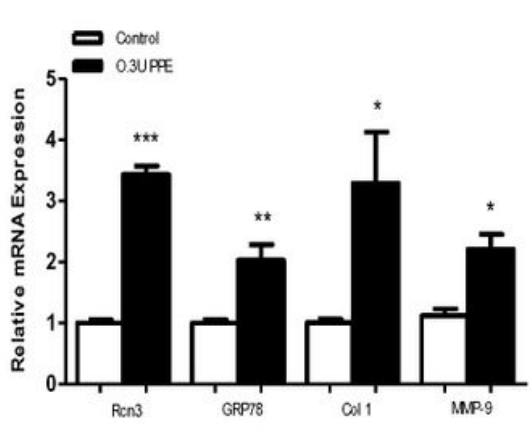

\section{Figure 3}

Rcn3 expression in lung tissues from the elastase-induced mouse model of emphysema. (A) Lungs after saline and PPE exposure at $0.2 \mathrm{U}, 0.3 \mathrm{U}$ and $0.4 \mathrm{U}$ /per mouse are compared in $\mathrm{HE}$-stained tissue sections; (B) The MLI analysis of lung sections after saline and PPE exposure $(n=20)$; (C) The PPE exposure induced the expressions of Rcn3, GRP78, Cleaved Caspase-3; $\beta$-Tubulin expression is the loading control, and the ratios to Tubulin expression were represented by graphs (D); (E) Quantitative RT-PCR analyses of the mRNA expressions of Rcn3, GRP78, Collagen 1 and MMP-9 in the lungs at 4 weeks after PPE (0.3U/per mouse) or saline treatment. The data are normalized to the RLP19 content; Data presented as mean $\pm S E M ;{ }^{*} p<0.05,{ }^{*} p<0.01,{ }^{* \star *} p<0.001$, versus sham group, $\# p<0.05$, versus $0.3 U$ PPE group. Bar size: $100 \mu \mathrm{m}$. 
Figure 4

A

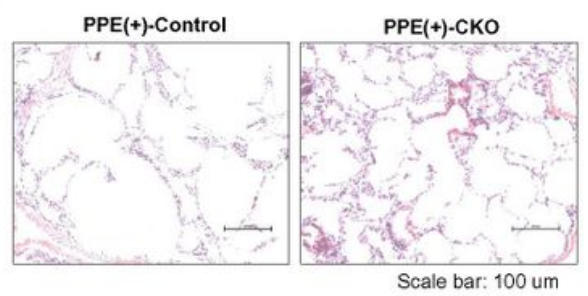

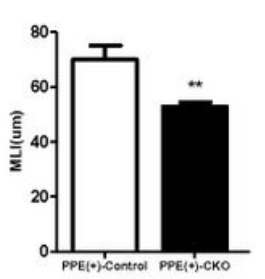

GRP78

C

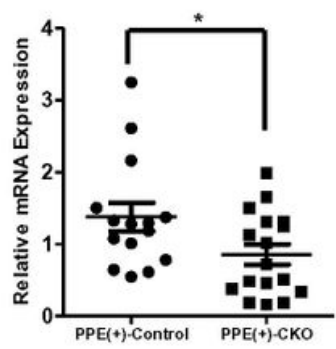

B
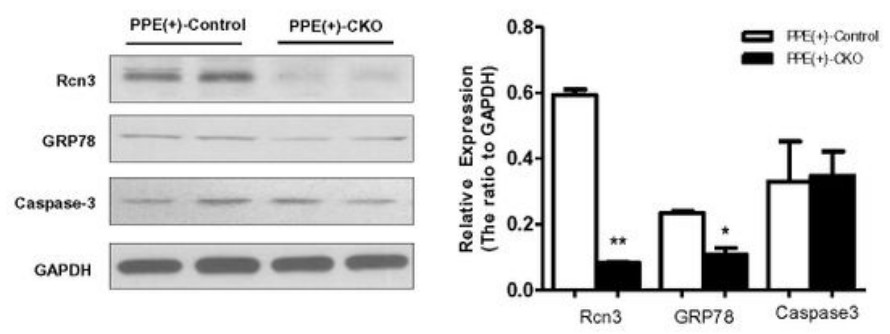

MMP-9
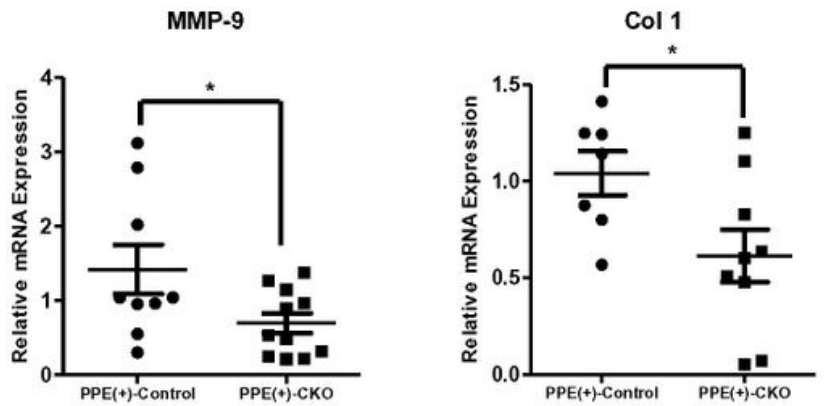

\section{Figure 4}

The selective deletion of Rcn3 in AEClls alleviated elastase-induced emphysema. (A) Photomicrography of pulmonary parenchyma stained with HE in two groups of mice; and the MLI analysis of lung sections in CKO mice and Control mice $(n=25)$; (B) The protein levels of Rcn3, GRP78 and cleaved caspase-3 are examined by Western blot analysis and the ratios to GAPDH presented by bar graph; (C) Qualitative RTPCR analysis of the mRNA of Rcn3, GRP78, MMP-9 and Collagen 1. The data are normalized to the RLP19 content; Data presented as mean \pm SEM; ${ }^{*} p<0.05,{ }^{*} p<0.01$, versus littermate control in the same treatment group. Bar size: $100 \mu \mathrm{m}$.

\section{Supplementary Files}

This is a list of supplementary files associated with this preprint. Click to download.

- AdditionalfileRR.docx 